\title{
Far Ultraviolet Emission from NGC 7009
}

\author{
R. C. Iping ${ }^{1},{ }^{2}$, G. Sonneborn ${ }^{2}$, S. R. McCandliss ${ }^{3}$ and Y-H. Chu ${ }^{4}$ \\ ${ }^{1}$ The Catholic University of America, Washington, DC 20064, USA \\ email: iping@milkyway.gsfc.nasa.gov \\ ${ }^{2}$ NASA/GSFC, Code 665, Greenbelt MD 20771, USA \\ ${ }^{3}$ Dept. of Physics and Astronomy, The Johns Hopkins Univ, Baltimore MD 21218, USA \\ ${ }^{4}$ Dept. of Astronomy, Univ. of Illinois, Urbana, IL 61801, USA
}

\begin{abstract}
The high-excitation planetary nebula NGC 7009 and its central star were observed with the Far Ultraviolet Spectroscopic Explorer (FUSE) through a $30 \times 30 \operatorname{arcsec}($ LWRS) aperture, and with the HIRS narrow slit $(1.25 \times 20 \mathrm{arcsec})$ to isolate the inner and outer parts of the nebula from the central star. The high-resolution $(15 \mathrm{~km} / \mathrm{s})$ nebular spectra $(910-1187 \AA)$ show strong emission from C III] and permitted transitions of He II N II, N III, S III, S IV, S VI and O VI, with stronger emission closer to the central star. In this paper we present results obtained on the spatial variation of these tracers of highly ionized gas. The hot central star of NGC 7009 is discussed in a separate paper by Sonneborn et al.
\end{abstract}

Keywords. planetary nebulae: individual (NGC 7009), ultraviolet: stars, techniques: spectroscopic, line: identification, space vehicles: FUSE

NGC 7009, the Saturn Nebula ( $d=0.86$ kpc, Fernandez 2004; angular size $\sim 16$ arcsec), is an elliptically shaped nebula with two ansae and fast, low-ionization emission regions (FLIERS). Diffuse X-ray emission was found with XMM (Guerrero et al. 2002), but whether the cause is shocks or collimated outflows is unknown. Here we report far-UV nebular spectra (910-1187 A, resolution $15 \mathrm{~km} \mathrm{~s}^{-1}$ ) of NGC 7009 made by the FUSE satellite. LWRS aperture spectra of the nebula plus central star were taken in Nov. 1999 (Iping \& Sonneborn 2001, 2003, Iping et al. 2002). Far-UV emission line spectra were obtained in June 2002 with the HIRS slit positioned 3" (Pos 1) and 8" (Pos 2) NE of the central star. A rich FUV nebular emission is present. Preliminary line IDs include He II, C II, C III], N I, N II, N III, S II, S III, S IV, S VI, Si IV, O I, O III, O VI and Al V (Fig. 1). Most are permitted transitions. Some features remain unidentified, and some are contaminated by overlying ISM atomic or $\mathrm{H}_{2}$ absorption (see Sonneborn et al. this volume). The O VI emission at Pos 1, close to the central star, is much stronger than at Pos 2 and indicates shocked gas with a temperature of $\sim 10^{6} \mathrm{~K}$, probably caused by the strong interaction of the fast wind from the central star $\left(v_{\infty} \sim 2450 \mathrm{~km} \mathrm{~s}^{-1}\right)$ with a slow wind expelled during the asymptotic giant branch phase.

\section{Acknowledgements}

Financial support has been provided in part by NASA grant NAG5-12347 to CUA .

\section{References}

Fernandez, R., Montero, H. \& Schwarz, H.E. 2004, ApJ, 603, 595

Guerrero, M.A., Gruendl, R.A. \& Chu, Y.-H. 2002, A\&A 387, L1

Iping, R.C. \& Sonneborn, G. 2001, BAAS Vol.34

Iping, R.C., Sonneborn, G., \& Chu, Y.-H. 2002, BAAS Vol 33, p.806

Iping, R.C. \& Sonneborn, G. 2003, IAU Symposium 209 2003, 209, p. 187 

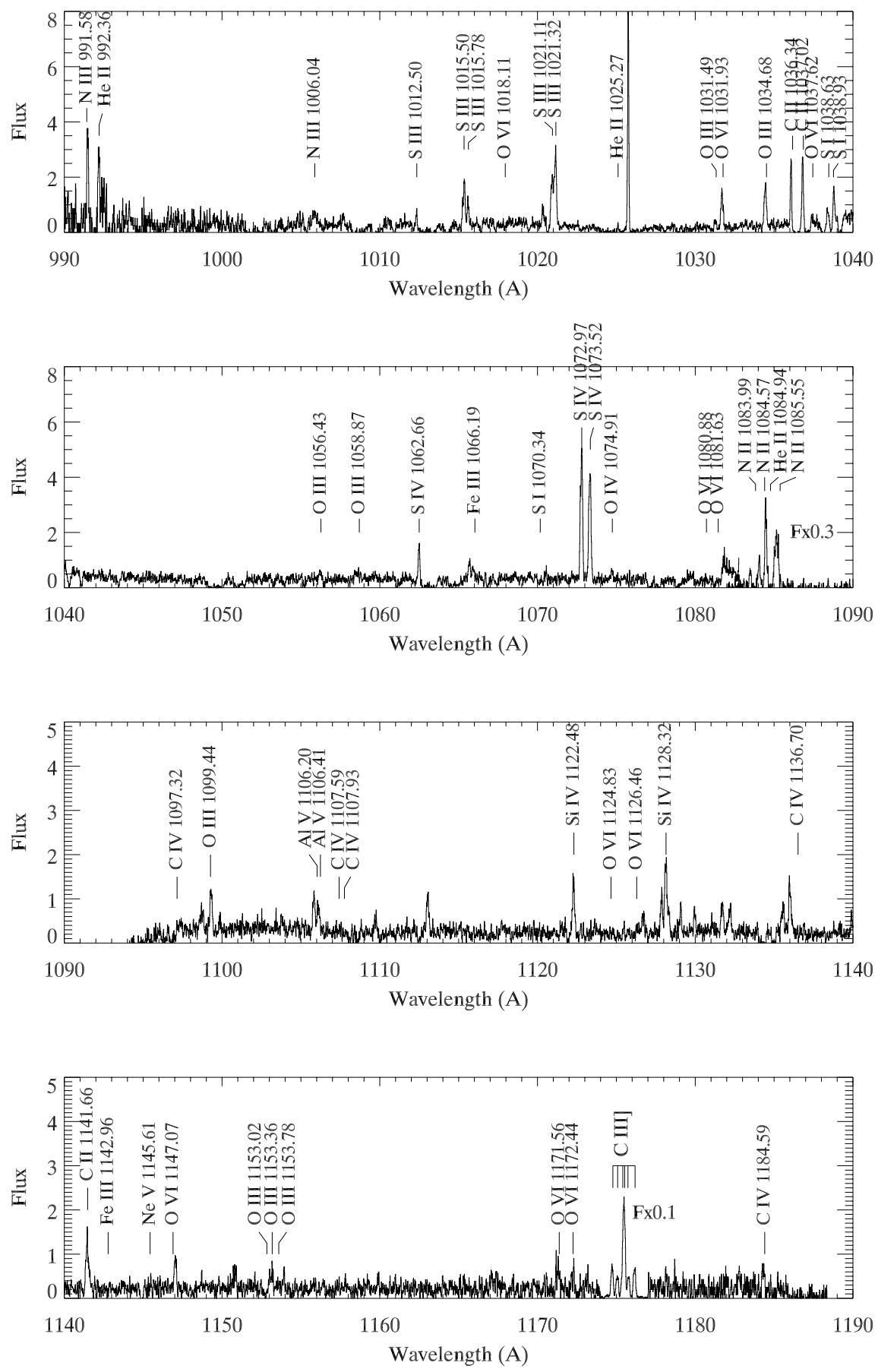

Figure 1. FUSE HIRS aperture (1.25x20 arcsec) LiF1 spectrum of the NGC 7009 nebula 3 arcsec NE of the central star (Position 1) from 990-1190 $\AA$ (SiC2b channel is used for 1082-1090 $\AA$ region). Fluxes are in units of $10^{-13} \mathrm{erg} \mathrm{cm}^{-2} \mathrm{~s}^{-1} \AA^{-1}$. The $1082-1090 \AA$ fluxes are scaled by 0.3 and $1174-1177 \AA$ by 0.1 to fit strong lines on the plot. 